\title{
Key Creative Features of Syntactic Design in English-Language Advertising Discourse
}

\author{
Elena N. Malyuga ${ }^{1} \&$ Barry Tomalin $^{2}$ \\ ${ }^{1}$ Peoples' Friendship University of Russia (RUDN University), Moscow, Russia \\ ${ }^{2}$ Glasgow Caledonian University London, London, UK \\ Correspondence: Elena N. Malyuga, Peoples' Friendship University of Russia (RUDN University), Moscow, 6 \\ Miklukho-Maklay Str., Russia. E-mail: en_malyuga@hotmail.com
}

Received: June 9, 2020 Accepted: July 10, 2020 Online Published: July 14, 2020

doi:10.5539/ijel.v10n5p145 URL: https://doi.org/10.5539/ijel.v10n5p145

\begin{abstract}
The study suggests that the patterns of syntactical arrangement should be viewed as indispensable creative features in designing advertising messages and postulates that three crucial aspects need to be addressed in order to comprehensively describe the specifics and benefits of a well-reasoned application of syntactic inventory of the English language for the purposes of constructing advertising texts. The three aspects - namely sentence type, message length and rhetorical tropes - are discussed at length from the discursive-pragmatic point of view and drawing on the texts of English-language advertisements of non-specific thematic affiliation. The study uses continuous sampling to ultimately make out the most commonly utilized sentence types, the most extensively preferred promotional message length, and the most frequently registered syntactic rhetorical tropes. The latter are further on filtered down to make up a list of seven syntax-driven rhetorical tropes of the most valid efficiency, followed by substantiation and analysis thereof. The study makes a number of conclusions suggesting that ad efficiency is strongly premised on the adequate comprehension and application of syntactic inventory, which implies selecting the most appropriate sentence type, considering the benefits of syntactic compression, positioning the arguments in the most advantageous way possible, and making use of the most expedient syntactic rhetorical tropes in order to garner the attention of a potential consumer, add an element of surprise and build up a more favorable attitude towards the product being advertised.
\end{abstract}

Keywords: advertising discourse, advertising text, syntax, rhetorical tropes

\section{Introduction}

The term "advertising" is derived from the Latin verb "advertere" literally meaning "pay attention to", specifically to a product or a service through some public advertisement (oral or written) (Hackley, 2010, p. 32). Advertising craft is an art of presenting something in a socially acceptable way allowing to persuade people to buy the advertised goods or services (Romanova \& Smirnova, 2019, p. 56). Given the efficiency of the appropriately arranged advertising discourse, it is quite understandable that it is used not only for promoting a brand but also as a tool of raising public awareness about social problems, as well as a centerpiece of political campaign strategies. Advertising professionals resort to an array of tools to enhance the message. More often than not, this discourse nowadays hinges on multimedia technologies which allow the advertisers to present information through various media (texts, audios and visuals). Advertising discourse may be characterized as a language of audial, visual and sensory impact which uses unconscious semantic meanings that resonate with people (Fennis \& Stroebe, 2015, p. 63). Advertising discourse aims to influence various social groups suggesting a particular way of thinking and lifestyle that are of current value. It is for this reason that advertising is one of the most exciting areas of activity of our day and age - in fact, it has become a means of communication molding people's attitude to products, companies, brands, lifestyles and social issues.

In other words, advertising is a large-scale phenomenon and given the complexity of the advertising discourse, we concur with LI's point of view stating that "one of the most efficient ways of understanding the meaning of an advertising message is to find out how this meaning was conveyed through language means" ( $\mathrm{Li}, 2019$, p. 520). Proceeding from this premise, this article aims to look into the advertising discourse in terms of analyzing its linguistic, particularly, syntactic aspect. Considering that syntax is an indispensable creative asset used in designing advertising messages, along with the lexis contained therein and its stylistic arrangement and 
rhetorical component, the theoretic study of this issue is essential to guarantee the full-fledged and comprehensive approach to analyzing the advertising discourse on the whole.

Advertising does not tolerate verbosity and complex syntactic turns, since by its nature it is supposed to be dynamic, i.e., it must call for action. As a rule, simple sentences prevail in text ads, which help make the advertising message more comprehensible an expressive. Phrases of a more complex syntactic structure need to be alternated with simple sentences. A short text will at all times be better perceived by the reader.

Apart from that, written advertising discourse is marked with a number of pragmatic peculiarities (including the desynchronized text production and understanding, the non-personal nature of the sender-recipient contact, the presence of a mediator between those represented by an advertising agency and the unilateral linguistic persuasion) which, coupled with its key orientation towards calling the recipient to action, form a distinct impact on the syntactic organization of advertising messages (Popova, 2017). This, in turn, rationalizes the relevance of the narrow focus on advertising syntax per se as a critical component of "the winning formula" of the advertising discourse.

Therefore, three research questions will be formulated as follows: (1) What sentences are the most effective when used in advertising slogans from the pragmatic point of view? (2) From the perspective of syntax, why are compressed messages more appropriate when it comes to comprehending and memorizing advertising slogans? (3) What syntactic rhetorical tropes can be considered the key advertising tools in English-language ads and why?

\section{Method}

Study material is represented by texts of English-language advertisements of non-specific thematic affiliation. The material was compiled by means of continuous sampling method using both printed and electronic textual and audiovisual sources. Overall, 319 examples were selected.

To analyze the singularities of the syntactic design of the collected material the structural, stylistic and pragmatic methods of analysis were used, which provided an insight into the nature of the formal syntactic composition of advertising texts and allowed us to describe the resulting specifics of the perception of advertising information by the recipient as intended by the creator.

In the study of the discursive-pragmatic potential of syntactic structures in English-language advertising texts, special attention was paid to the interaction of the social and the psychological in the language of advertising. The complex nature of the advertising discourse implies the need to carefully consider the entire set of diverse factors that influence the generation and interpretation of meanings. The methodology of discourse analysis, aimed at studying the discursive and pragmatic features of the syntactic structures of interest to this study, covers screening of the material, its processing, segmentation, classification, comparison, and statistical description with due consideration of the type of discourse being analyzed. Of interest to this study are the syntactic resources and structures chosen by the author in creating promotional texts for specific purposes (creating a certain effect, conveying a certain meaning, motivating a certain contingent of potential consumers, etc.).

In accordance with the objectives of the study, the material was screened through targeted continuous sampling of individual sentences, slogans, headings and texts of various sizes, which are part of English-language advertising discourse. During processing, the selected material was classified by type of sentences, volume (compressed vs extended message), as well as syntactic tools of rhetorical influence. Further interpretation required an interpretive analysis of the selected material in view of the intentional message of the author of the advertising text, its functional load, as well as the possible resultant effect, significant from the point of view of the goals of the advertising discourse.

\section{Advertising Discourse and the Language of Advertising: Theoretical Background}

By the day, the problems of advertising discourse garner attention of ever more language scholars, who in their studies identify the means used by advertisers to create promotional headlines, texts and slogans aimed at boosting potential customers' purchasing activity.

In the digital age, advertising discourse plays a very important role and is one of the fastest growing ones because of its relevance. Particular emphasis in the research of advertisements is given to the linguistics of the text and its description from the point of view of the "text-discourse" fundamental paradigm. Advertising texts evolve in response to discursive changes, and in the face of a vast number of studies scrutinizing advertising discourse the issue still holds sway because of the persistently progressing market of advertising services.

Advertising is a special type of communication designed to provide information about products, services, etc. in 
order to create demand for a particular group of goods (Altstiel et al., 2018), most commonly effectuated on commercial terms (Knoll, 2016). Advertisements are shared through various types of media-in the press, on television, radio, the Internet, etc. This paper puts emphasis on the study of online advertising, since this particular type of advertising has recently gained traction among consumers. The proliferation of the Internet, wireless communications, mobile devices, and mobile Internet is helping drive up visibility among users and, hence, potential consumers. The more repeatedly a user-consumer goes online, the greater their exposure to an advertisement for a particular product (Světlík, 2017). By implication, advertising affects the user's consciousness and consolidates within it, thus making it more likely for them to purchase this particular product in their next shopping tour.

Advertising discourse is a variety of institutional discourse, which is considered as a special clichéd form of communication between people who may not know each other, but who must communicate as per social norms. Communication within the institutional discourse is hinges on a person's social status and his position as a community member (Malyuga, 2019). As a rule, institutional discourse involves eradicating all personal descriptors, i.e. it is assumed that a person follows the norms and rules of the society they happen to be a part of.

Due to its extensive nature, advertising discourse is found in all types of institutional discourse, since the topics covered within the scope of advertising activity are nothing short of unlimited. Advertising penetrates into all spheres of human life, and its purpose lies not only in attracting the attention of the audience, but also in prodding people into a particular action. In this case, the participants in the institutional discourse are advertising agents and, accordingly, people who turn to them, i.e., clients (the advertiser and the consumer) (Todorova, 2018, p. 298).

One of the defining attributes of advertising discourse is that it draws on a variety of functional styles: modern advertising messages extensively leverage functional styles such as conversational, public, professional and business styles. Very often professional jargon is found in the texts of advertising messages, and by the same token the boundaries between individual styles are becoming ever more blurred.

Advertising discourse is treated as a combination of an advertising text and extralinguistic factors, and is defined as a completed message bearing an exhaustively focused pragmatic attitude (drawing attention to the subject of advertising), endowed with the distinctive features of spoken language and written text, as well as a set of semiotic (para- and extralinguistic) means.

Advertising can only reach its goals provided that the process of its compilation is rigorously contemplated so as to appreciate and take a satisfactory account of the intricacies of the human psyche. In composing an advertising message, it is customary to apply the AIDA formula (standing for "attention - interest - desire - action") developed in 1986 by the American advertiser Elmer Lewis. This seemingly ingenious model incorporates the fundamental functions of any advertising message: attract attention, arouse interest, develop it into a strong desire bordering on yearning, then prompt into action.

An advertising text, by virtue of its brevity, is an example of the most effective application of language means. And it is here that the connection of advertising discourse with pragmalinguistics becomes clearly visible, since the latter actually dictates which grammatical patterns, lexical units, stylistic techniques or syntactic constructions will be ultimately used.

Most often, advertising is not created with the sole purpose of conveying information, but is intended as a tool helping create a vivid promotional image for the recipient. To achieve this, various types of stylistic tools are used in advertising texts (irony, metaphor, hyperbole, etc.).

The language of advertising performs two communicative functions - to inform and convince. It must be literate, comprehensive, specific and purposeful, allowing one to appreciate in what way the advertised object is actually different from the similar counterparts. At the same time, the language of advertising texts should be evidence-based (convincing), logical in terms of both form and content. Distinctiveness, whimsy and the captivating nature of advertising texts highlight the artistic side of such narratives, and syntax is one of the tools helping support and illuminate the various artistic functions of advertising discourse.

In contrast to the concept of "text", which was relatively recently established within the linguistic science, the concept of "syntax" is more traditional. Since ancient times, the language was said to incorporate three integral components, namely phonetics, vocabulary and grammar. Syntax arises at the next stage of the hierarchy as part of grammar, and is first given prominence in the 3rd century BC among the Stoics to denote the logical structure

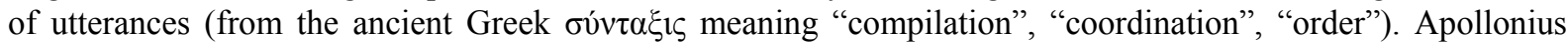
Diskol (2nd century AD) considers linguistic phenomena as the subject of syntax - the connection between of 
words and their forms in a sentence (Miller, 2016).

Since then, interpretations of syntax have been changing depending on the era and the views of the scholars, with various schools and trends in semiotics, philosophy and linguistics coming along and focusing on the diverse aspects and issues of language systems in general and syntax in particular. According to Roberts (2018), syntax constitutes part of grammar that deals with units longer than the word, i.e., phrases and sentences, whereas the key difference between a sentence and a word is a more complex hierarchical structure, the capacity of a sentence to accumulate unlimited added complexity, as well as the direct bond between the sentence and the utterance, a pronounced communicative nature.

Syntax in its modern-day interpretation is closely intertwined with stylistics, since all the main functional stylistic and expressive properties of language units of different levels are manifested within the margins of a certain syntactic context (Malyuga et al., 2019). The presence of a wide range of logical, communicative and expressive syntactic tools makes syntax the most universal tool for the successful implementation of communication in different types of discourse, including advertising discourse.

The study singled out three key syntactic characteristics that form a core of the syntactic nature of the English-language advertising discourse: 1) the predominance of a certain type of sentences in advertising texts; 2) syntactic compression and specifics of argumentative elements arrangement within a sentence; 3) syntactic rhetorical tropes.

\section{Results and Discussion}

\subsection{Types of Sentences}

As material analysis demonstrated, English-language advertising discourse utilizes all sentence types: (1) declarative sentences, i.e., presenting a message in a declarative form (e.g., "There's no wrong way to eat a Reese's"); (2) imperative sentences designed to straightforwardly motivate the potential consumer to purchase goods/services (e.g., "Put a TicTac in your mouth and get a bang out of life"); (3) exclamatory sentences normally adding positive connotation, as well as making the intended recipient perceive information in the positive light (e.g., "Gotta have my pops!", "I go cukoo for Cocoa Puffs!"); (4) interrogative sentences, creating the illusion of a dialogue with the recipient, provoking them to stop and reflect on a possible answer and a problem which is relevant to the recipient on the whole: (4a) general questions (e.g., "Curious. Do you dare?"); (4b) special or wh-questions (e.g., "Sears. Where else?”); (4c) choice questions (e.g., "Long \& Foster. Thinking or sailing?"); (4d) disjunctive or tag questions (e.g., "Starburst. Life is juicy, isn't it?"); (4e) questions to the subject (e.g., “Arby's. Who's on board?”).

Also within the realm of the interrogative-sentence-approach in advertising is the strategy whereby a relevant question is immediately supported with a catchy answer. Constructions containing questions and responses in advertising texts are borrowed from colloquial syntax and are used to attract the attention of the consumer to the content of the message, encourage them to think independently (excluding questions devoid of specific content). Such constructions make the presentation sound unforced and casual. With this technique, a potential question of a consumer is somewhat predicted so that an immediate answer could be provided on the spot, as in: "What do the beautiful people drink when they get thirsty? Same as everyone else. Sprite." "How many licks does it take to get to the Tootsie Roll Center of a Tootsie Pop? The world may never know. Tootsie Pop."

Despite greater structural diversity of the latter (interrogative) type, declarative sentences have become the most common in English-language advertising discourse (Orlova \& Tsagolova, 2017), as they allow to attract the audience's attention and add expressiveness to a slogan. For instance: "Hook up with a buddy online, get your own meal plan and easy recipes, or chat with a dietitian. C'mon, if I can be a big loser, there's nothing stopping you."

In this example, the advertiser uses imperative sentences as low-pressure commands persuading the recipient to perform a certain action - to join the weight loss program. In some cases, imperative sentences are more preferable, as they are more convincing and may become the very incentive that at times aggressively, but mostly efficiently, urges the recipient to purchase the advertised product/service.

Special emphasis needs to be put on exclamatory sentences as an exhaustively effective means of coloring the advertising text with the expressive power. The phenomenon of expressivity is based on several groups of psychological laws relating, on the one hand, to the expression of emotions and feelings, and on the other, to perception. The linguistic mechanism behind expressivity is mainly a deviation from stereotypes in the use of language units of various levels.

The emergence of expressivity as a characteristic feature of an advertising text depends on the properties of the 
communicative situation: the intentions of the author of the text, the presumptions of the reader (i.e., the initial knowledge and ideas that they have at the time the text is being perceived), as well as the linguistic and extralinguistic (social) context. The main components that make up the concept of "expressiveness" in advertising are intensity, emotional load, imagery and evaluative power (Curtis et al., 2017, p. 59). That being said, imagery can be considered as a fundamental creative asset of exclamatory sentences found in advertising discourse, since it allows to mold a new, more vivid idea about the advertised product, which is why it becomes an important tool in the advertiser's arsenal. Exclamatory sentences, therefore, can be considered as a stand-alone creative means that help embellish the ad and attract the attention of the potential consumer: "Butterfinger: Nobody better lay a finger on my Butterfinger!" "Trix Cereal: Silly Rabbit, Trix are for kids!" "Kelogg's Frosted Flakes: They'rrrre GR-R-REAT!" "Crest: Look Ma, no cavities!"

\subsection{Syntactic Compression and the Arrangement of Arguments Within a Sentence}

The length of a sentence is a critical criterion of text readability - the longer the sentence, the lower the sentence readability. According to a recent study by the University of Michigan, a regular text, regardless of its subject-matter and terminological density, with the average sentence length of eight or fewer words, is considered "very readable", while a message containing 29 or more words is going to be deemed "very hard to read". Meanwhile, the "standard" or ideal median is 17 words (Collins-Thompson, 2014, pp. 99-101).

As for an advertising text, one has to factor in the target audience of such a message which is geared primarily toward arresting attention and verbosity - i.e., a longer sentence within such a text would compromise this goal and weaken audience interest (Daugherty et al., 2018, p. 189). This premise is further corroborated by the analysis of the material used in the present study, where the average length of all examined texts was estimated at 9.8 words.

According to the results of the recent study on the subject of the short-term memory capacity, the optimum number of symbols for memorizing ay information should not exceed nine (Britt et al., 2017, p. 112). Therefore, of all the structural elements, an advertising message often contains only the slogan and the brand name conveying one unique idea. The most common examples of super-short phrases are advertisements of elite brands for which their name speaks for itself, for example, Dior, Chanel, Louis Vuitton, Salvatore Ferragamo and the like.

In the advertising discourse, another crucial factor determining the success of a message is its arguments positioning with the key argument located in the heading, the evidence argument placed in the main text (body) and the generalizing argument exposed in the echo-phrase. Any types of sentences can be used as arguments in advertising discourse: "Berkshire Hathaway. Trusting our heritage" (the key argument in the form of as a declarative sentence is in the heading). "Why trust the purchase or sale of a luxury home to anyone else?" (the main text features a reasoning argument in the form of as an interrogative sentence). "To find sales professionals go to Berkshire hathaways.com" (the echo-phrase is an imperative sentence). Appeal is usually exploited as an argument, being associated with profit and economy, belonging to a particular group, benefit for health, as well as leisure.

\subsection{Syntactic Rhetorical Tropes}

Any proposition contained in an advertising text may be expressed in various ways, and in any particular situation one of those ways is going to prove most efficient in terms of its impact on the audience. Consequently, the expressive method of a message may, from the rhetorical standpoint, be more important than its propositional content. This premise places an emphasis on the use specific rhetorical tropes as a key characteristic of English-language advertising discourse.

A rhetorical trope is traditionally defined as an artistic digression (Hart \& Daughton, 2015, p. 19) and emerges when the utterance deviates from the expected one, with the meaning of the utterance not discarded as pointless or erroneous. The deviation happens on the level of the form, rather than the content, and matches the pattern utilized in an array of contexts.

Just like the aesthetic objects on the whole, a rhetorical figure allows to add an element of surprise to a familiar concept. Such element, introduced through linguistic digression, underpins what consumer behaviorists call "the incongruity of perceptions" (De Mooij, 2019). Rhetoric's key contribution is that it explains how certain types of textual structures generate the "incongruity" effect in the mind of consumers when they are faced with an advertising text.

The use of rhetoric figures in advertising activity is always a premeditated and productive strategy whose goal is to attract the potential customer's attention in the context of various circumstances of the recipient's contact with 
this advertisement. The advertiser always considers a simple fact when creating an advertising text - a person who enters into "contact" with the ad, has the right to ignore it or make minimum effort to process its message and meaning. As consumers are not obliged to begin, continue or finish reading the heading or the main text of an ad, an important function of rhetorical figures lies in motivating the potential recipient to pay attention to it. The text's digression from a standard, common and expected one is one of the tools intended to arrest attention.

Tropes also produce what the semiotics scholar Roland Barthes referred to as "the pleasure of decoding" (Wiseman, 2016, p. 98) - it is a reward derived from processing skillfully arranged characters. This, in turn, corresponds to the argument assuming that language incongruity (digression) can cause a pleasant extent of excitement (Mohanty \& Ratneshwar, 2016, p. 449). Thus, the positive nature and efficiency of a skillfully presented digression implies that the highly figurative language of advertising should create a more positive attitude to advertising than the literary advertising language (Danilina et al., 2019). This argument is further supported by evidence suggesting that the figurative language of advertising is always more memorable. At this point the study shall consider the most common syntactic rhetorical tropes used by advertising copywriters to grasp the attention of potential consumers.

As per study results, the main functional-stylistic and expressive syntactic tools and their functions in the texts of English-language advertising can be summarized as follows.

Syntactic anaphora - the use of an expression that depends specifically upon an antecedent expression; repetition of the same syntactic constructions. Functions: (1) laying emotional emphasis on any part of the statement; (2) creating the effect of alternating events; (3) creating the effect of obsession, fixation. Example: "EVN. Always here, always with you."

Antithesis - a sharp juxtaposition of concepts, provisions, images, conditions, interconnected through a common construction or internal meaning. Functions: (1) providing emotional comparison of opposites; (2) appealing to the logic of negation; (3) creating the effect of expressiveness, persuasiveness and vividness of counterarguments. Example: "Google: Know more, waste less."

Asyndeton-deliberate omission of conjunctions. Functions: (1) transferring the meaning of swiftness, dynamism of action; (2) transferring the meaning of a rapid change of scenery, impressions, actions; (3) creating the effect of the unity of enumeration. Example: "Gatorade: Float like a Butterfly, Sting like a Bee, 1/2 the calories, all the G."

Gradation - arrangement of parts of an utterance related to a single subject so that each subsequent part is more saturated, more expressive or impressive than the previous one. Functions: (1) intensifying the logical-semantic arguments; (2) enforcing emotional perception; (3) creating the effect of engagement of increasing persuasive power. Example: "Rice Crispies: Snap! Crackle! Pop!"

Inversion - changing the meaning of a word by placing a sentence in a syntactically unconventional place. Functions: (1) emphatic highlighting of a word or phrase; (2) creating the effect of surprise, attracting attention. Example: "IBM: Such is the moment that all greats traverse."

Lexical repetition-intentional repetition in a small part of the text of the same word or speech structure. Functions: (1) laying logical and/or emotional emphasis on any part of the statement; (2) creating the effect of alternating events; (3) creating the effect of obsession, fixation. Example: "Milwaukee River Keeper: A clean river is a fun river."

Oxymoron - a witty combination of conflicting concepts (syntax-wise, usually represented through an adjective and a noun), a paradox. Functions: (1) allocating objects, problems, contradictions; (2) creating the effect of surprise; (3) creating the illusion of solving an insidious problem. Example: "Maxwell House: Alone together."

Parallel constructions - arrangement in adjacent parts of a text of speech elements that are identical or similar in terms of syntactical and semantical structure. Functions: (1) comparing parallel elements; (2) providing a logical argument; (3) suggestion an emotion-driven argument or sentiment. Example: "Chevrolet: Eye it, try it, buy it!"

Antimetabole (less commonly referred to as antimetathesis) - repetition of words found on the left side of a complex sentence in its right side with a change in their syntactic functions. Functions: (1) emphatic highlighting of the entire utterance; (2) creating the effect of expressiveness and vividness. Example: "Lexus: Drive to live, not live to drive."

Parcellation - deliberate division of a coherent text into several segments independent in terms of punctuation and intonation. Functions: (1) slowing down the speech tempo; (2) highlighting details; (3) creating the effect of surprise, contrast; (4) creating the effect of a long-acting action or actions. Example: "Asana: Get Asana. Get 
results. Do great things together."

Polysyndeton - the intentional use of excess conjunctions. Functions: (1) slowing down the speech tempo; (2) enhancing the role of each word; (3) creating the effect of the unity of enumeration. Example: "Skinlabs: Night Repair makes your skin clean. And smooth. And young."

Refrain-repetition of a portion of a text, word, or speech structure, usually in a poem. Functions: (1) laying logical and/or emotional emphasis on the main topic of the text; (2) creating the effect of an obsession, fixation. Example: "WholeFoods: Healthy food bless us but junk food mess us."

Rhetorical question - a question put in the form of an affirmation that does not require an answer. Functions: (1) focusing on a problem; (2) suggesting an emotional argument, persuasion due to the obvious nature of the answer; (3) creating a humorous, ironic effect. Example: "Dr. Pepper: What's the worst that could happen?"

Aposiopesis - an intentional break of a statement accompanied by a violation of syntactic relations. Functions: (1) creating the effect of emotional speech; (2) suggesting emotional involvement; (3) suggesting speculation, a hidden logical argument. Example: "Ricola: She's (cough) just a friend."

Chiasmus - cross-arrangement of words and phrases in the first and second parts of the utterance. Functions: (1) highlighting the second emphatic part of the utterance due to an unexpected pause in front of it; (2) creating a humorous, ironic effect. Example: "Hyposwiss Bank: You give us your money. And we give it back to you."

Ellipsis - an intentional omission of words that are not essential to the meaning of an expression. Functions: (1) accelerating or simplificating the speech; (2) suggesting emotional involvement through speculation; (3) creating a humorous, ironic effect. Example: "Safari: Tomorrow's skills, today."

Epiphora-repetition of words within consecutive subordinate clauses. Functions: (1) endowing a part of the statement with an emphatic stress; (2) creating the effect of obsession, fixation; (3) transferring the idea of speculation. Example: "I'm a Pepper, he's a Pepper, she's a Pepper, we're a Pepper. Wouldn't you like to be a Pepper, too? Dr. Pepper."

Thus, the syntax provides a variety of tools that help enhance the communicative and psychological impact of the text and expand its stylistic and expressive capabilities within the texts of advertising discourse. According to study results, some of the rhetorical tropes are more frequently encountered in English-language ads, and these will be discussed below.

As the syntax of advertising texts is distinctly succinct, which is at times similar to the brevity of the telegraphic style, one of the most conspicuous syntactic singularities of the advertising discourse is the use of elliptical constructions. Ellipsis occurs when there is a lexical gap in the text the meaning of which cannot be deduced from the context. In other words, either one or both principal parts of the sentence are not formally present, or are present in the syntactic environment only, or are just implied by the speech situation. A case in point is Suzuki's slogan reading "Every day vehicles that aren't". In this case, the reader is supposed to fill in the gap by "completing" the sentence and reinstating it structural and semantic integrity-Every day vehicles that aren't every day (aren't ordinary, aren't mundane, aren't commonplace). This is how a special degree of figurativeness is created, helping attract the recipient's attention and give them the possibility to enjoy their own ability to decipher the message ("the pleasure of decoding"). This very technique was used by Michelin who entered the market with the slogan "A lot of tires cost less than Michelin. That's because they should". It is the implied meaning conveyed through the elliptical constructions (omissions) that makes such advertising stand out, which, in turn, is a sign of its potential.

In the case of parallel constructions, another frequent device in advertising discourse, the structure of one sentence (or its part) recurs in another sentence which in a way "copies" its syntactic composition resulting in an identical or similar arrangement of speech elements in adjacent parts of the text (Booij \& Audring, 2017, p. 277). The purpose of using a parallel construction is to endow the message with a certain rhythm. Parallel syntactic constructions are often accompanied with phonetic stylistic devices typical of a poem and allow to create short, memorable and unique advertising texts. For instance, parallel constructions in English-language advertising discourse are often combined with anaphor, as this trope combination allows to efficiently retain and register the emphasized repeated element in the reader's memory: "Maybe she's born with it. Maybe it's Maybelline." "You never had it so easy. Your tires never had it so good."

A type of parallel constructions that came to be known in rhetoric as antimetabole is also featured in the study materials as one of the most common. Antimetabole combines expressive elements that mirror each other, i.e., two parts of an utterance repeat one another but in reverse order (Cui \& Zhao, 2014, p. 58). Thus, "Stop static before static stops you"-a slogan describing a fabric softener-may be divided in two structural parts. In the 
first part the noun "static" is an (action) object of the verb "stops", while the noun "static" in the second part functions as an action subject for the verb "stops". Also noteworthy is the use of alliteration as an additional attention-arresting element, since in advertising discourse various rhetoric tropes are often integrated within one slogan, which allows to multiply the effect and add vividness to an advertising text.

Equally popular among advertising copywriters is the technique of inversion. The use of inversion adds to creating a conspicuous image. Rearrangement, which disrupts the normal word order, either entails the change in the logical content of the sentence or emotionally intensifies the entire statement, as is the case, for instance, with Adidas' slogan "Impossible is nothing".

Next in the study results is the technique of parcellation - a syntactic structure involving an intentional decomposition of segments that are independent punctuation-wide, yet connected with each other intonationally, semantically and graphically (Borchers \& Hundley, 2018, p. 109). The point indicates a syntactic break. Parceling structures are a characteristic feature of motivational slogans - this technique is often encountered in advertising texts creating a certain rhythm, emphasizing a redundant part of an utterance and simplifying its structure as exemplified by Sony's slogan "Make. Believe".

An adjacent trope is the one commonly referred to as "segmented structure". In this syntactic construct, the first part is designed to name an object or phenomenon in order to evoke a certain idea (expressed by a noun in the nominative case). In the subsequent text, the object or phenomenon receives a second designation in the form of a pronoun: "Meow Mix. Tastes so good, cats ask for it by name."

Lastly, there are rhetorical questions, which are popular among advertisers for an obvious reason- they help to put pressure on the reader, while playing down and decreasing the aggressiveness at the same time. A case in point is the rhetorical question in the slogan for facsimile machines by Hewlett-Packard which reads "Don't you have something better to do?" Instead of a direct statement, the advertisement is based on a question, thus covering up and enhancing the call to action. Clearly, the rhetorical question in this case should be formulated so as to let the potential customer correct the meaning on their own, replacing the one loosely connected with the expression with the one which better matches the context of interpretation (Theodorakis et al., 2015, p. 15). This is why rhetorical questions serve as catalysts for the thinking process making the readers reflect on further actions and often contain implicit positive answers. The idea is that should the recipient ponder the question, they will find the answer in the same advertisement: "HSBC. Thinking about changing your mortgage lender?" "IBM. Is your business ready to launch a new business?"

\section{Conclusion}

Original and effective combinations of language means found in advertising texts present a very popular object of modern-day research. At times, advertising goes beyond the existing language standards, shaping new textual visualizations and models of verbal communication in its search for maximum impact effectiveness, which only later become an integral element of linguistic culture. As was shown in the theoretical review on the subject, advertising texts continue to attract the attention of linguists, which is reflected in the increased number of studies addressing the various issues associated with this phenomenon. Recent decades have been marked with the growing scholastic interest centered around the patterns of language use in various areas of advertising communication, and the diverse aspects of advertising texts. There is an extensive number of studies scrutinizing English-language advertising discourse, which is only natural, given the fact that it is the media texts in English that dominate the global information flow, both in volume and in terms of influence.

This paper has dwelled upon the notion of the advertising text considered as a prosaic or poetic text containing a hidden or open call to action, usually aimed at increasing demand and viewed as one of the main means of attracting the target audience. The language tools and speech strategies used in advertising largely determine how successful the product is going to be. In this regard, linguists focus extensively on an all-encompassing study of this concept.

This article looked into the principal syntactic characteristics of English-language advertising discourse, the analysis of which reveals that as far as the singularities of the syntactical structure are concerned, the language of advertising has more in common with the spoken language, i.e., a structurally simplified style. The study was based on revealing the three key syntactic characteristics: (1) the predominance of certain (imperative) sentences in advertising texts, (2) the preferred design of advertising texts committed towards syntactic compression and a specific composition of arguments within a sentence and (3) the abundance of syntactic rhetorical techniques.

The main findings of the study can be presented in the following way: (1) success of an advertising slogan depends not only on the correct selection of words, but also on the syntactic organization of the ad; (2) although 
all types of sentences are present in English-language advertising slogans, imperative sentences are the most common and pragmatically effective; (3) an advertising message is better perceived and memorized when it is constructed as a short text subject to syntactic compression; (4) advertising text efficiency depends on the positioning of the arguments in its syntactic structure, and the most effective model is where the key argument is located in the heading, the evidence argument is placed in the main text, and the generalizing argument is exposed in the echo phrase; (5) syntactic rhetorical tropes are an important advertising tool, since they are more memorable and can attract the attention of a potential consumer, adding an element of surprise and creating a more positive attitude towards the product as a whole; (6) English advertising texts typically contain such syntactic tropes as ellipsis, parallel constructions, antimetabole, inversion, parcellation, segmented structures, and rhetorical questions.

\section{Acknowledgments}

This paper was financially supported by the Ministry of Education and Science of the Russian Federation on the program to improve the competitiveness of Peoples' Friendship University of Russia (RUDN University) among the world's leading research and education centers in 2016-2020.

\section{References}

Altstiel, T., Grow, J., \& Jennings, M. (2018). Advertising creative: Strategy, copy, and design. London: Sage Publications.

Booij, G., \& Audring, J. (2017). Construction morphology and the parallel architecture of grammar. Cognitive Science, 41, 277-302. https://doi.org/10.1111/cogs.12323

Borchers, T., \& Hundley, H. (2018). Rhetorical theory: An introduction. Waveland Press.

Britt, M. A., Rouet, J. F., \& Durik, A. M. (2017). Literacy beyond text comprehension: A theory of purposeful reading. Routledge. https://doi.org/10.4324/9781315682860

Collins-Thompson, K. (2014). Computational assessment of text readability: A survey of current and future study. International Journal of Applied Linguistics, 165(2), 97-135. https://doi.org/10.1075/itl.165.2.01col

Cui, Y., \& Zhao, Y. (2014). Translation of rhetorical figures in the advertising discourse: A case study. International Journal of Society, Culture \& Language, 2(2), 57-67.

Curtis, T., Arnaud, A., \& Waguespack, B. P. (2017). Advertising effect on consumer emotions, judgements, and purchase intent. Asian Journal of Business Research, 7(2), 57-73. https://doi.org/10.14707/ajbr.170037

Danilina, E. A., Kizyan, E. E., \& Maksimova, D. S. (2019). Euphemisms in advertising discourse: putting on a positive face and maintaining speech etiquette. Training, Language and Culture, 3(1), 8-22. https://doi.org/10.29366/2019tlc.3.1.1

Daugherty, T., Hoffman, E., Kennedy, K., \& Nolan, M. (2018). Measuring consumer neural activation to differentiate cognitive processing of advertising: revisiting Krugman. European Journal of Marketing, 52(1-2), 182-198. https://doi.org/10.1108/EJM-10-2017-0657

De Mooij, M. (2019). Consumer behavior and culture: Consequences for global marketing and advertising. Sage Publications Limited.

Fennis, B. M., \& Stroebe, W. (2015). The psychology of advertising. Psychology Press. https://doi.org/10.4324/9781315681030

Hackley, C. (2010). Advertising. Sage Publications Limited. https://doi.org/10.4135/9781446260807

Hart, R. P., \& Daughton, S. (2015). Modern rhetorical criticism. Routledge. https://doi.org/10.4324/9781315663555

Knoll, J. (2016). Advertising in social media: A review of empirical evidence. International Journal of Advertising, 35(2), 266-300. https://doi.org/10.1080/02650487.2015.1021898

Li, S. (2019). Expanding the scope for study on global English-language advertising. World Englishes, 38(3), 519-534. https://doi.org/10.1111/weng.12428

Malyuga, E. N. (2019). Emergent trends in English scientific discourse: issues of research relevance and linguistic identity. Tomsk State University Journal of Philology, 58, 52-70. https://doi.org/10.17223/19986645/58/4

Malyuga, E. N., Poliakova, N., \& Tomalin, B. (2019). Syntactic constructions featuring multifunctional sentence components in the language of modern business media. International Journal of English Linguistics, 9(4), 
58-69. https://doi.org/10.5539/ijel.v9n4p58

Miller, J. (2016). Introduction to English syntax. Edinburgh University Press.

Mohanty, P., \& Ratneshwar, S. (2016). Visual metaphors in ads: The inverted-U effects of incongruity on processing pleasure and ad effectiveness. Journal of Promotion Management, 22(3), 443-460. https://doi.org/10.1080/10496491.2016.1154924

Orlova, S. N., \& Tsagolova, A. S. (2017). Linguacultural characteristics of English-language advertising discourse. Issues of Applied Linguistics, 28, 84-95. https://doi.org/10.25076/vpl.28.08

Popova, K. V. (2017). Strategy of persuasion in social advertising. Issues of Applied Linguistics, 28, 66-73. https://doi.org/10.25076/vpl.28.06

Roberts, I. (2018). Diachronic and comparative syntax. Routledge. https://doi.org/10.4324/9781315310572

Romanova, I. D., \& Smirnova, I. V. (2019). Persuasive techniques in advertising. Training, Language and Culture, 3(2), 55-70. https://doi.org/10.29366/2019tlc.3.2.4

Světlík, J. (2017). Integrating online advertising into integrated marketing communications. Marketing Identity, $5(1), 206-215$.

Theodorakis, I. G., Koritos, C., \& Stathakopoulos, V. (2015). Rhetorical maneuvers in a controversial tide: Assessing the boundaries of advertising rhetoric. Journal of Advertising, 44(1), 14-24. https://doi.org/10.1080/00913367.2014.930679

Todorova, R. (2018). Knowledge, reality and imagination in advertising discourse. Knowledge International Journal, 22(1), 297-301.

Wiseman, M. B. (2016). The ecstasies of Roland Barthes. Routledge. https://doi.org/10.4324/9781315538006

\section{Copyrights}

Copyright for this article is retained by the author, with first publication rights granted to the journal.

This is an open-access article distributed under the terms and conditions of the Creative Commons Attribution license (http://creativecommons.org/licenses/by/4.0/). 